\title{
La participación española en los programas europeos: análisis estructural del área de salud del 6. Programa Marco
}

\author{
José Luis Ortega*, Isidro F. Aguillo**
}

\begin{abstract}
Resumen: El objetivo de este trabajo es describir la participación española en el área temática de salud del $6 .^{\circ}$ Programa Marco. Para ello se pretende realizar un análisis estructural de la red de colaboraciones entre las diversas entidades que participan en tales proyectos además de una descripción al nivel regional, sectorial e individual del grado de participación y retorno obtenido. Los resultados muestran que la participación española se apoya en el sector administración, de ámbito estatal, concretamente en el CSIC, el CNIO, los hospitales y las universidades. Se concluye que España presenta una posición periférica en el área temática de salud, cuya participación se sustenta básicamente en el CSIC.
\end{abstract}

Palabras clave: Cienciometría, análisis de redes sociales, Programas Marco, colaboración científica.

\section{Spanish participation in EU research programmes: structural analysis of the health-related thematic area of the 6th Framework Programme}

Abstract: The aim of this work is to describe the participation of Spanish institutions in the 6th Framework Programme's thematic area on bealth. It presents a structural analysis of the collaboration network among the different organizations that participate in these projects, as well as a description of the degree of participation and of the results obtained, on a regional, sectorial, and individual basis. The results show that Spanish participation lies primarily with the governmental sector, especially on a national level, with the principal involvement concentrated in CSIC, CNIO, hospitals, and universities. The article concludes that Spain is on the periphery of the health-related thematic area, with participation largely undertaken by CSIC.

Keywords: Scientometrics, social network analysis, Framework Programmes, scientific collaboration.

* Vicepresidencia de Investigación Científica y Técnica, CSIC, Madrid. Spain. Correo-e-: jortega@ orgc.csic.es.

** Laboratorio de Cibermetría, CCHS-CSIC, Madrid, España. Correo-e: isidro.aguillo@cchs.csic.es. Recibido: 28-7-09; aceptado: 10-12.09. 


\section{Introducción}

El avance experimentado en los últimos años en materia de computación ha permitido el estudio en profundidad de las relaciones estructurales en sistemas complejos como la Web (Barabasi y Albert, 1999), la difusión de enfermedades (Pastor-Satorras y Vespignani, 2001) y las cadenas tróficas (Polis y Strong, 1996). La actividad científica también está caracterizada como un sistema complejo en el que diversos agentes (empresas, administración, universidades, etc.) interactúan en un entorno sujeto a múltiples variables. El uso de análisis estructurales en este ámbito ha permitido estudiar los fenómenos de colaboración científica en las publicaciones científicas (Newan, 2001; Barabasi y otros, 2002; Wagner and Leydesdorff, 2005), redes de citación entre artículos (Small, 1999) y revistas (Leydesdorff, 2004) o las relaciones entre patentes (Valverde y otros, 2007).

El sistema de I+D europeo se apoya en los Programas Marco (PM) para potenciar la investigación científica en el marco de la Unión, además de ser un vehículo para la cohesión del Espacio Europeo de Investigación. Estos programas asumen la colaboración como una característica fundamental en la actividad científica europea en la que los proyectos deben desarrollarse por diversas organizaciones pertenecientes a diferentes países y sectores. Este entorno interrelacionado implica una gran oportunidad para entender como se establecen estas relaciones, qué actores participan en la red y cual es su rol, y como opera esta red en la mejora del sistema de I+D de la Unión Europea.

Trabajos anteriores han analizado las redes de colaboración en el marco de los programas europeos de investigación. Breschi y Cusmano (2004) estudiaron las alianzas estratégicas (joint ventures) del $3 .^{\circ}$ y $4 .^{\circ}$ Programa Marco, observando un fenómeno de atracción preferencial entre ambas convocatorias. Barber y otros (2006) estudiaron las propiedades estructurales desde el $2 .^{\circ}$ hasta el 5. ${ }^{\circ}$ Programa Marco, observando propiedades de libre escala, distribuciones potenciales (power laws) y alto grado de agrupamiento. Roediger-Schulga y Dachs (2006) encontraron diferencias significativas en dos áreas temáticas del $6^{\circ}$ Programa Marco. Estos detectaron que mientras en el área de telecomunicaciones participaban más socios industriales y el presupuesto era elevado, en el de agricultura estaban más presentes la investigación pública y contaba con menos presupuesto. RoedigerSchulga y Barber (2007), utilizando los mismos datos, visualizaron el 5. ${ }^{\circ}$ Programa Marco, mostrando que el núcleo central de la red estaba ocupado por grandes organizaciones científicas. Finalmente, cabe destacar los trabajos de Gusmao (2000; 2001) donde se analiza la participación de los países de la Unión, destacando sus características particulares.

En España existen diversos trabajos que, aunque no han analizado los proyectos de investigación desde un punto de vista estructural, si han descrito patrones de colaboración. En este caso cabe destacar el trabajo de Fernández y otros (1992) sobre los proyectos realizados entre el Consejo Superior de Investigaciones Científicas (CSIC) y entidades latinoamericanas. En Finlandia destaca el 
estudio sobre la participación de este país en el 5. ${ }^{\circ}$ Programa marco (Uotila y otros, 2004) junto a los estudios en la República Checa (Albrecht y Vaněček, 2008), en Suecia (Arnold y otros, 2008).

\section{Objetivos}

El objetivo de este trabajo es describir la participación española en el área temática de salud del 6. ${ }^{\circ}$ Programa Marco (6PM). Para ello se pretende realizar un análisis estructural de la red de colaboraciones entre las diversas entidades que participan en tales proyectos. Se busca conocer qué características fundamentales presenta la red española, cuáles son sus principales agentes, cuál es el papel de los diferentes tipos de organizaciones y cuál es su contexto en el ámbito internacional.

\section{Metodología}

Se modelizó una red de 2.132 organizaciones participantes en 601 proyectos de investigación pertenecientes al área temática de "Ciencias de la vida, Genómica y Biotecnología para la Salud" del 6PM. Estos datos se obtuvieron a través del Centro de Desarrollo Tecnológico Industrial (CDTI) en diciembre de 2007. Esta red representa las relaciones de colaboración entre distintas instituciones a través de diferentes proyectos europeos. A partir de esta red se detectaron las instituciones pertenecientes a España, obteniéndose 106 instituciones de las cuales 83 estaban conectadas entre sí.

Para la visualización de la red se utilizó el programa Pajek 1.02 (Nooy y otros, 2005). El algoritmo de Kamada-Kawai (1991) fue usado para energizar dicha red. Para el análisis estructural y la extracción de indicadores se utilizó Ucinet 6 (Borgatti y otros, 2002). Los siguientes indicadores fueron usados:

- Grado de centralidad $(k)$ : mide el número de líneas incidentes a un nodo (Freeman, 1979). Este indicador puede ser normalizado por el número total de nodos en la red. El Grado de centralidad permite detectar organizaciones que tienen un alto grado de colaboración con diferentes instituciones, mostrando una alta actividad en los programas de investigación.

- Grado de intermediación (Freeman, 1980): mide la capacidad de un nodo de mantener conectados aquellos nodos que no están conectados directamente con otros. Su normalización es calculada como el porcentaje sobre el número total de nodos en la red. Desde un punto de vista cienciometrico, esta medida nos permite detectar concentradores (hubs) u organizaciones que atraen diferentes socios a los programas europeos de investigación. 


\section{Resultados}

\subsection{Regiones}

La tabla I muestra la distribución de organismos que participan en el área temática de salud del 6PM por Comunidades Autónomas. Las regiones que más organizaciones aportan son Cataluña (30,19\%), Madrid (17,92\%) y Andalucía $(10,38 \%)$. Uno de los problemas de los proyectos europeos, es que estos son firmados por el rector o presidente de un organismo de investigación, en vez de por el propio investigador. Esto ocasiona que los datos no estén desagregados al nivel de instituto o centro de investigación. Este hecho es especialmente significativo cuando tratamos con instituciones de la administración central, como los Ministerios y el CSIC, cuya actividad repercute a todo el país. Por esta razón, en la tabla I se ha creado una entrada denominada "Estatal" que recoge a estos organismos. La tabla II lista los nueve organismos pertenecientes a esta categoría.

Destaca la situación de Cataluña, la cual casi duplica el número de instituciones de Madrid y se aleja considerablemente de las anteriores. Si observamos esta misma distribución según el porcentaje de subvención que recibe cada región,

TABLA I

Distribución de Organizaciones por Comunidades Autónomas

\begin{tabular}{l|c|c|c|c|c|c}
\hline \multicolumn{1}{c|}{ Regiones } & Org. & \% Org. & $\begin{array}{c}\text { \% } \\
\text { Subvención }\end{array}$ & $\begin{array}{c}\text { Media de } \\
\text { proyectos }\end{array}$ & $\begin{array}{c}\text { Media } \\
\text { de socios } \\
\text { españoles }\end{array}$ & $\begin{array}{c}\text { Media } \\
\text { de socios } \\
\text { internacionales }\end{array}$ \\
\hline Cataluña & 32 & 30,19 & 1,23 & 3,38 & 3,46 & 47,31 \\
\hline Madrid & 19 & 17,92 & 0,41 & 1,89 & 1,82 & 28,37 \\
\hline Andalucía & 11 & 10,38 & 0,14 & 1,27 & 1,30 & 17,27 \\
\hline Estatal & 9 & 8,49 & 1,26 & 12,22 & 11,56 & 133,67 \\
\hline País Vasco & 8 & 7,55 & 0,16 & 2,00 & 1,88 & 29,63 \\
\hline Valencia & 8 & 7,55 & 0,36 & 3,38 & 3,00 & 52,50 \\
\hline Asturias & 4 & 3,77 & 0,05 & 1,75 & 1,75 & 30,00 \\
\hline Castilla y León & 4 & 3,77 & 0,07 & 2,67 & 2,67 & 63,33 \\
\hline Navarra & 4 & 3,77 & 0,14 & 2,25 & 2,25 & 35,50 \\
\hline Galicia & 3 & 2,83 & 0,10 & 2,00 & 2,00 & 19,00 \\
\hline Aragón & 1 & 0,94 & 0,03 & 2,00 & & 46,00 \\
\hline Baleares & 1 & 0,94 & 0,01 & 1 & 2 & 38 \\
\hline Cantabria & 1 & 0,94 & 0,01 & 1,00 & & 7,00 \\
\hline Murcia & 1 & 0,94 & 0,00 & 1,00 & 5,00 & 24,00 \\
\hline TOTAL & 106 & 100 & 3,97 & 3,27 & 4,02 & 44,67 \\
\hline
\end{tabular}




\section{TABLA II}

\section{Organismos Estatales}

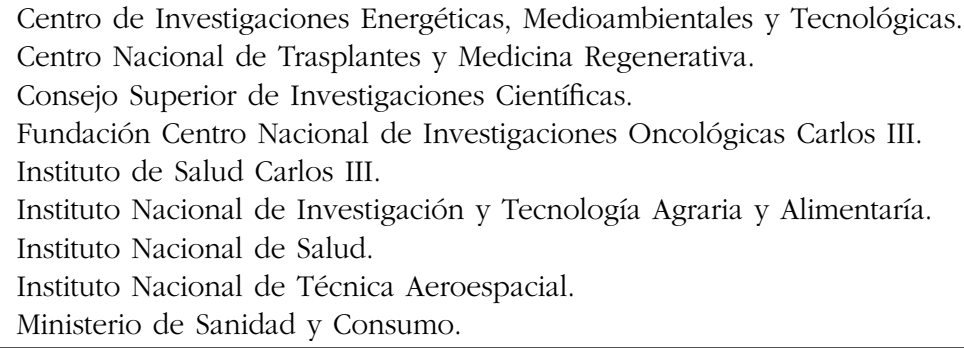

son las instituciones estatales las que mayor porcentaje de subvención reciben $(1,26 \%)$, seguidas muy de cerca por Cataluña $(1,23 \%)$ y Madrid $(0,41 \%)$. Sorprende que en el conjunto de todo el país apenas se llegue a alcanzar el 4\% del total de fondos del área temática de salud del 6PM. Este patrón es similar en la distribución de la media de proyectos por cada región, siendo las organizaciones estatales $(12,22)$ las que más participan en proyectos junto a Cataluña $(3,38)$ y Valencia $(3,38)$. Al igual que en los proyectos, los organismos estatales son los que poseen un promedio más elevado de socios nacionales $(11,56)$ e internacionales $(133,67)$. A continuación, la región con más socios nacionales es Murcia (5) - este valor debe considerarse con cautela ya que esta región tan sólo aporta una única organización- y Cataluña $(3,46)$. Con respecto a los socios internacionales, son Castilla y León $(63,33)$ y Valencia $(52,5)$ las regiones que más socios foráneos tienen. Un hecho interesante es señalar el escaso promedio de socios nacionales $(4,02)$ que presentan las entidades españolas en contraposición a la cantidad de socios internacionales $(44,67)$ con los que colaboran.

\subsection{Tipo de organizaciones}

La tabla III muestra la distribución de organizaciones según su tipo. Esta clasificación se realizó según la clasificación institucional por sectores del Manual de Frascati (OEDC, 2003). Las organizaciones españolas que más predominan en el área temática de salud del 6PM son las empresas (34,91\%), universidades $(32,08 \%)$ y las administraciones públicas (25,47\%). Sin embargo, si nos atenemos al porcentaje de subvención que reciben, son los organismos públicos $(1,85 \%)$ los que mayor porcentaje obtienen, seguido de las universidades (1,33\%) y las empresas $(0,69 \%)$. En cuanto a la media de proyectos en que participan son las administraciones las que más participan $(6,15)$, junto a las universidades $(3,12)$ y las empresas $(2,78)$. Si observamos el promedio de socios, siguen siendo las administraciones las que mayor número de socios poseen tanto a nivel nacional $(6,21)$ como internacional $(84,15)$. 
TABLA III

Distribución por tipo de organización

\begin{tabular}{l|c|c|c|c|c|c}
\hline Tipo de organizaciones & Org. & \% Org. & $\begin{array}{c}\text { \% } \\
\text { Subvención }\end{array}$ & $\begin{array}{c}\text { Media de } \\
\text { proyectos }\end{array}$ & $\begin{array}{c}\text { Media } \\
\text { de socios } \\
\text { nacionales }\end{array}$ & $\begin{array}{c}\text { Media } \\
\text { de socios } \\
\text { internacionales }\end{array}$ \\
\hline Administración Pública & 27 & 25,47 & 1,85 & 6,15 & 6,21 & 84,15 \\
\hline Universidades & 34 & 32,08 & 1,33 & 3,12 & 3,75 & 45,18 \\
\hline Empresas & 37 & 34,91 & 0,69 & 1,78 & 2,77 & 21,00 \\
\hline $\begin{array}{l}\text { Organizaciones sin fi- } \\
\text { nes de lucro }\end{array}$ & 8 & 7,55 & 0,11 & 1,13 & 2,40 & 18,75 \\
\hline Total & 106 & 100 & 3,97 & 3,27 & 4,02 & 44,67 \\
\hline
\end{tabular}

\subsection{Organizaciones}

La tabla IV muestra el listado de las 10 primeras organizaciones por el porcentaje de subvención. Este listado está encabezado por el Consejo Superior de Investigaciones Científicas (CSIC) con 0,75\%, seguido por la Universidad Autónoma de Barcelona con un $0,24 \%$ y el Centro Nacional de Investigaciones Oncológicas (CNIO) con también 0,24\%. Según el número de proyectos en que participan, es el CSIC el que interviene en más proyectos (63) junto al CNIO (16)

TABLA IV

Distribución de Organizaciones ordenadas por el porcentaje de subvención

\begin{tabular}{l|c|c|c|c|c}
\hline \multicolumn{1}{c|}{ Organización } & $\begin{array}{c}\text { \% } \\
\text { Subvención }\end{array}$ & $\begin{array}{c}\text { Coordi- } \\
\text { nador }\end{array}$ & Proyectos & $\begin{array}{c}\text { Socios } \\
\text { nac. }\end{array}$ & $\begin{array}{c}\text { Socios } \\
\text { inter. }\end{array}$ \\
\hline $\begin{array}{l}\text { Consejo Superior de Investigaciones Cien- } \\
\text { tíficas }\end{array}$ & 0,75 & 6 & 63 & 41 & 444 \\
\hline Universidad Autónoma de Barcelona & 0,24 & 2 & 14 & 9 & 160 \\
\hline $\begin{array}{l}\text { Centro Nacional de Investigaciones Onco- } \\
\text { lógicas }\end{array}$ & 0,24 & & 16 & 6 & 171 \\
\hline Universidad de Valencia & 0,16 & 2 & 10 & 17 & 154 \\
\hline Hospital Clínico y Provincial de Barcelona & 0,13 & & 15 & 13 & 278 \\
\hline $\begin{array}{l}\text { Institut d'Investigacions Biomèdiques Au- } \\
\text { gust Pi i Sunyer }\end{array}$ & 0,13 & 3 & 9 & 5 & 107 \\
\hline Universidad Pompeu Fabra & 0,12 & 1 & 9 & 5 & 79 \\
\hline Universidad Autónoma de Madrid & 0,12 & & 8 & 4 & 109 \\
\hline Fundació Centre de Regulació Genomica & 0,11 & & 4 & 1 & 50 \\
\hline Instituto Nacional de Salud & 0,11 & & 13 & 13 & 257 \\
\hline
\end{tabular}


y al Hospital Clínico y Provincial de Barcelona (15). Si nos fijamos en el número de proyectos que coordinan, considerándolo como un indicador de prestigio, vuelve a ser el CSIC el que más proyectos coordina (6), seguido por el Institut d'Investigacions Biomèdiques August Pi i Sunyer (3). Ateniéndonos al número de socios, el CSIC es el organismo que más socios posee tanto nacionales (41) como internacionales (444), le sigue la Universidad de Valencia con respecto a socios nacionales (17) y el Hospital Clínico y Provincial de Barcelona a nivel internacional (278).

\subsection{Análisis estructural}

La figura 1 muestra la red de los 83 socios participantes en los proyectos de salud del 6PM. El tamaño de cada nodo muestra el porcentaje de financiación que cada organismo ha obtenido de su participación en el programa. El grosor de cada arco muestra el número de proyectos conjuntos en que participan dos organizaciones. La forma corresponde al tipo de organización, siendo el triangulo para la administración pública, el circulo para las universidades, cuadrado para las empresas y el rombo para las instituciones sin ánimo de lucro. Cada organización fue clasificada según los criterios del Manual de Frascati (OEDC, 2003).

Lo primero que podemos ver es la posición central del CSIC $(k=41)$, que como hemos visto en la tabla IV es el organismo que más socios posee, además de ser el que más subvención recibe. Junto a éste está la Universidad de Valencia $(k=17)$ y el Hospital Clínico y Provincial de Barcelona $(k=13)$ como los organismos que más colaboran en la red nacional. En el gráfico se puede apreciar que no existe agrupación por criterios regionales, únicamente se aprecia un grupo de instituciones catalanas en la parte superior izquierda y una triada aislada de instituciones vascas también en el margen superior izquierdo. La figura también muestra que los centros de la administración poseen de media mayor centralidad ( $k=5,85)$ que el resto de tipo de instituciones, mientras que las Organizaciones sin ánimo de lucro son las que menor centralidad media poseen $(k=1,12$, seguidas por las Empresas $(k=1,63)$. Si observamos el grado de intermediación, entendida como el grado de atracción que poseen determinadas organizaciones para atraer socios a la red, vuelven a ser el CSIC $(0,34)$ y la Universidad de Valencia $(0,1)$ los organismos que más organizaciones atraen, seguidos esta vez por el Instituto Nacional de Salud $(0,06)$.

\section{Conclusiones y discusión}

El análisis de la participación española en el área temática de salud del 6PM nos ha permitido mostrar un retrato de los principales agentes en la investigación biomédica en España. Pensamos que esta muestra constituye la élite de la investigación en salud ya que el bajo grado de éxito (18\%) en las solicitudes hace 


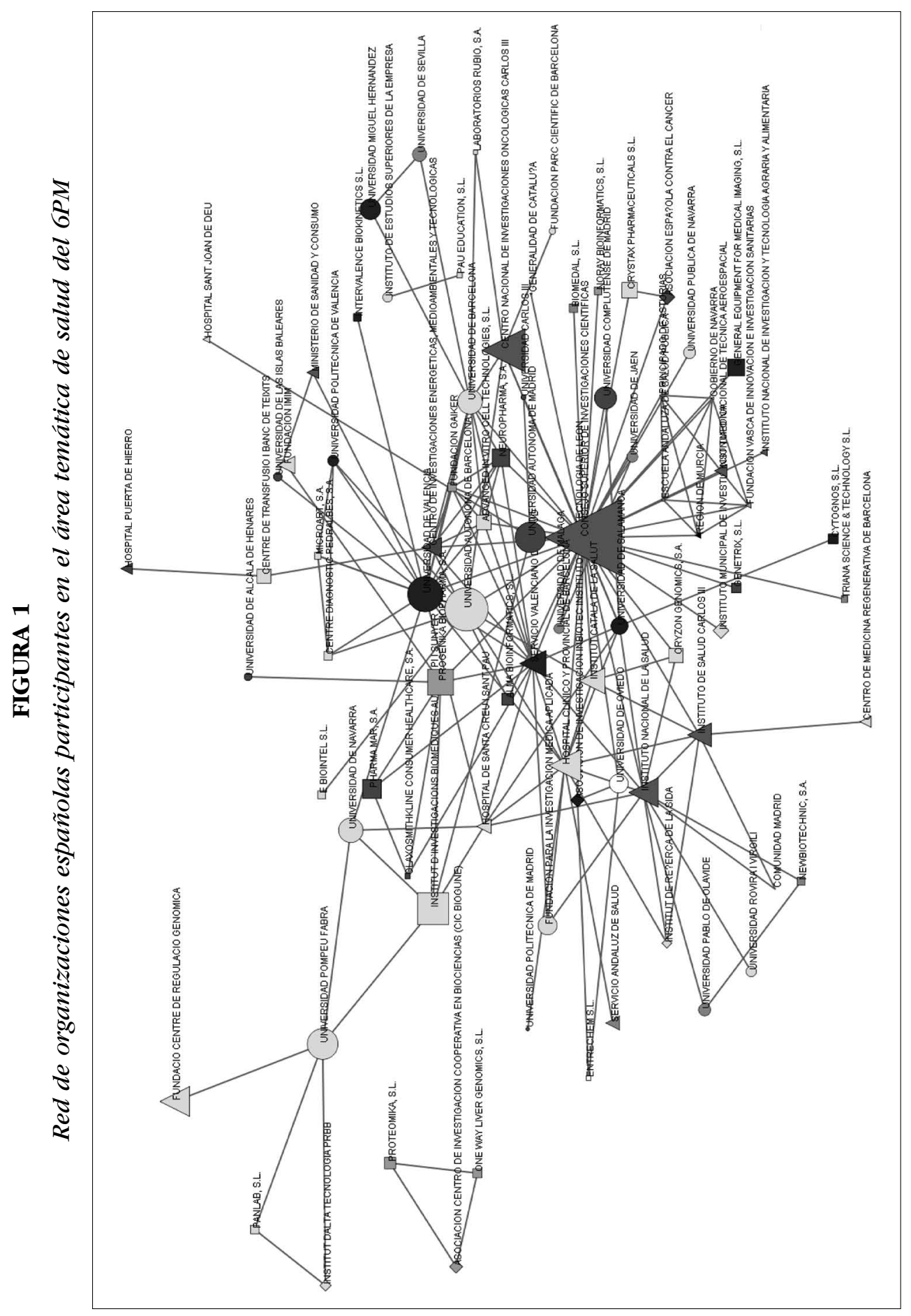


que la participación en los programas marco sea considerado como un indicador de excelencia científica en cada país (Comisión Europea, 2008). Estudios previos (Ortega y Aguillo, 2010, en prensa) han mostrado que a pesar de que España es el $6 .^{\circ}$ país por el número de instituciones participantes, es la 9. ${ }^{a}$ por la cantidad de retorno que obtiene por su participación. Esto indica que la participación de las organizaciones españolas no obtiene el mismo retorno en proporción a su participación. Otro indicador, el porcentaje de participación/liderazgo de proyectos sitúa a España en el puesto $13 .^{\circ}$, lo que confirma la posición periférica de la investigación española en los Programas Marco europeos, al menos en el área temática de salud.

El análisis interno nos muestra que las principales regiones por participación son Cataluña, Madrid y Andalucía, aunque por porcentaje de subvención es Valencia la tercera comunidad autónoma. Hemos decidido escindir en la categoría estatal a las organizaciones cuyo ámbito de actuación no se limita a una única región como son organismos de la administración central y el CSIC. Creemos que esta categoría evita sobredimensionar la región de Madrid cuando contabilizamos la I+D realizada por organismos de ámbito estatal cuya sede esta en dicha comunidad. Esta categoría estatal es la que mayor porcentaje de subvención recibe por lo que podemos concluir que estos centros son las entidades más importantes en esta área temática. Estos resultado contrastan con el estudio bibliométrico de Méndez-Vásquez y otros (2008), donde surge Madrid como la región más productiva seguida por Cataluña y Andalucía, debido a esta concentración de organismos estatales en la comunidad de Madrid.

En cuanto al tipo de organización, en el caso de España se aprecia una alta presencia del sector privado (34,91\%), aunque el grado de participación es bastante bajo (1,78 proyectos por organización). Sin embargo, el sector de la administración es el que mayor retorno obtiene (1,85\%) además de mayor participación $(6,15$ proyectos por organización) pese a tener una presencia discreta $(25,47 \%)$. La alta presencia de empresas puede deberse al incremento del sector biotecnológico (Gravalos y otros, 2002). Sin embargo, el hecho de que la administración obtenga la mayor proporción de subvención se debe a que ésta es la que más participa de media en más proyectos, por lo que el retorno es mayor. Podemos concluir que mientras el sector Empresas es el que más organizaciones aporta, es el sector Administración el que más participa en distintos proyectos. Ortega y Aguillo (2008) ya percibieron esta situación a nivel europeo.

Los principales actores españoles por el grado de subvención son el CSIC, la Universidad Autónoma de Barcelona y el Centro Nacional de Investigaciones Oncológicas, mientras que por participación se incluyen también el Hospital Clínico y Provincial de Barcelona y el Instituto Nacional de Salud. El análisis estructural ha confirmado esta posición ya que estas mismas instituciones son las que mayor centralidad presentan. Análisis bibliométricos también coinciden en identificarlas como las más productivas (Méndez-Vásquez y otros, 2008). Esto nos permite afirmar que estos centros son el eje en que descansa la investigación biomédica española. 


\section{Agradecimientos}

Agradecemos al Departamento de Programa Marco de I+D del Centro para el Desarrollo Tecnológico Industrial (CDTI) por su apoyo y suministro de los datos relativos al área temática de salud del 6. ${ }^{\circ}$ Programa Marco.

\section{Bibliografía}

Albrecht, V., y Vaněček, J. (2008): Assessment of Participation of the Czech Republic in the EU Framework Programmes. Praga: Technology Centre of the Academy of Sciences of the Czech Republic.

Arnold, E.; Astrom, T.; Boekholt, P.; Brown, N.; Good, B.; Holmberg, R.; Meijer, I., y Van der Veen, G. (2008): Impact of the Framework Programme in Sweden. Estocolmo: VINNOVA.

Barabasi, A. L., y Albert, R. (1999): Emergence of Scaling in Random Networks. Science, vol. 286(5439), 509-512.

Barabasi, A.; Jeong, H.; Neda, Z.; Ravasz, E.; Schubert, A., y Vicsek, T. (2002): Evolution of the social network of scientific collaborations. Physica A, vol. 311 (3-4), 590-614.

Barber, M. J.; Krueger, A.; Krueger, T., y Roediger-Schulga, T. (2006): The Network of European Research and Development Projects. Physical Review E (Statistical, Nonlinear, and Soft Matter Physics), 73 (3), 036132.

Borgatti, S. P.; Everett, M. G., y Freeman, L. C. (2002): Ucinet for Windows: Software for Social Network Analysis. Harvard, MA: Analytic Technologies.

Breschi, S., y Cusmano, L. (2004): Unveiling the texture of a European Research Area: Emergence of oligarchic networks under the EU Framework Programmes. International Journal of Technology Management, vol. 27 (8), 747-72.

Cabo, P. G. (1999): Industrial participation and knowledge transfer in joint R\&D projects International Journal of Technology Management, vol. 18 (3-4), 188-206.

Clavería, L. E.; Guallar, E.; Camí, J.; Conde, J.; Pastor, R.; Ricoy, J. R.; Rodríguez-Farré, E.; Ruiz-Palomo, F., y Muñoz, E. (2000): Does Peer Review Predict the Performance of Research Projects in Health Sciences? Scientometrics, vol. 47 (1), 11-23.

Comisión Europea (2008): FPG Final Review: Subscription, Implementation, Participation. Brussels: Research Directorate-General.

Fernández, M. T.; Agis, A.; Martín, A.; Cabrero, A., y Gómez, I. (1992): Cooperative research projects between the Spanish National Research Council and Latin-American institutions. Scientometrics, vol. 23 (1), 137-148.

Freeman, L. C. (1979): Centrality in networks: I. conceptual clarification. Social Networks, vol. 1, 215-239.

Freeman, L. C. (1980): The gatekeeper, pair-dependency, and structural centrality. Quality and Quantity, vol. 14, 585-592.

Gravalos, E.; Garcia, A., y Barnes, N. (2002): Policy influences on innovation strategies of small and medium enterprises in the agrochemical, seed and plant biotechnology sectors. Science and Public Policy, vol. 29 (4), 277-285 (9). 
Gusmao, R. (2000): Developing and Using Indicators of Multilateral S\&T Cooperation for Policy Making: The Experience from European Research Programmes. Scientometrics, vol. 47(3), 493-514

Gusmao, R. (2001): Research networks as a means of European integration. Technology in Society, vol. 23, 383-393.

Kamada, T., y Kawai, S. (1991): A general framework for visualizing abstract objects and relations. ACM Transactions on Graphics, vol. 10 (10), 1-29.

Kruskal, W. H., y Wallis, W. A. (1952): Use of ranks in one-criterion variance analysis. Journal of the American Statistical Association, vol. 47 (260), 583-621.

Lepori, B.; Van den Besselaar, P.; Dinges, M.; Van der Meulen, B.; Poti, B.; Reale, E.; Slipersaeter, S., y Theves, J. (2007): Indicators for comparative analysis of public project funding: concepts, implementation and evaluation. Research Evaluation, vol. 16 (4), 243-255.

Leydesdorff, L. (2004): Clusters and maps of science journals based on bi-connected graphs in Journal Citation Reports. Journal of Documentation, vol. 60 (4), 371-427.

Méndez-Vásquez, R. I.; Suñén-Pinyol, E.; Cervelló, R., y Camí, J. (2008): Mapa bilbiométrico de España 1996-2004: biomedicina y ciencias de la salud. Medicina Clínica, vol. 130 (7), 246-253.

Newman, M. E. (2001): Scientific collaboration networks. I. Network construction and fundamental results. Physical Review E, vol. 64 (1), 016131.

Nooy, W. de; Mrvar, A., y Batagelj, V. (2005): Exploratory Social Network Analysis with Pajek. Cambridge, UK: Cambridge University Press.

OECD (2003): Frascati Manual 2002. Paris: OECD Publishing.

Ortega, J. L., y Aguillo, I. F. (2008): Network collaboration in Life sciences 6th Framework Programme: a visual approach. In: $10^{\text {th }}$ International Conference on Science and Technology Indicators, Vienna.

Ortega, J. L., y Aguillo, I. F. (2008): Network collaboration in the $6^{\text {th }}$ Framework Programmes: country performance in the health program. Scientometrics (en prensa).

Pastor-Satorras, R., y Vespigniani, A. (2001): Epidemic Spreading in Scale-Free Networks. Physical Review Letters, vol. 86 (14), 3200-3203.

Polis, G. A., y Strong, D. R. (1996): Food Web Complexity and Community Dynamics. The American Naturalist, vol. 147 (5), 813-846.

Roediger-Schulga, T., y Barber, M. J. (2007): RED collaboration networks in the European Framework Programmes: Data processing, network construction and selected results. Maastricht: United Nation University.

Roediger-Schluga, T., y Dachs, B. (2006): Does technology affect network structure? A quantitative analysis of collaborative research projects in two specific EU programmes. Maastricht: United Nation University.

Small, H. (1999): Visualizing science by citation mapping. Journal of the American Society for Information Science, vol. 50 (9), 799-813.

Uotila, M.; Kutinlahti, P.; Kuitunen, S.; Loikkanen, T. (2004): Finnish Participation in the EU Fifth Framework Programme and Beyond. Helsinki: Finnish Secretariat for EU R\&D,

Valverde, S.; Solé, R. V.; Bedau, M. A., y Packard, N. (2007): Topology and evolution of technology innovation networks. Physical Review E, vol. 76 (5), 056118.

Wagner, C. S., y Leydesdorff, L. (2005). Network structure, self-organization, and the growth of international collaboration in science. Research Policy, vol. 34 (10), 1608-1618. 